\title{
Breast Cancer Detection by Analyzing the Volatile Organic Compound (VOC) Signature in Human Urine
}

\section{Judit Giró Benet ( $\nabla$ jgirbene@uci.edu )}

University of California Irvine (UCI), Center for Embedded Cyber-physical Systems (CEPS)

\section{Minjun Seo}

University of California Irvine (UCI), Center for Embedded Cyber-physical Systems (CEPS)

\section{Michelle Khine}

University of California Irvine, Department of Biomedical Engineering

\section{Fadi Kurdahi}

University of California Irvine (UCI), Center for Embedded Cyber-physical Systems (CEPS)

\section{Josep Gumà Padró}

Rovira i Virgili University (URV), South Catalonia Institute of Oncology (IISPV), Sant Joan University Hospital (HUSJ)

\section{Antonio Pardo Martínez}

Department of Biomedical Engineering and Electronics, Universitat de Barcelona (UB), Spain

\section{Research Article}

\section{Keywords:}

Posted Date: February 2nd, 2022

DOI: https://doi.org/10.21203/rs.3.rs-1296727/v1

License: (c) (i) This work is licensed under a Creative Commons Attribution 4.0 International License.

Read Full License 


\title{
Breast cancer detection by analyzing the volatile organic compound (VOC) signature in human urine
}

\author{
Judit Giró Benet ${ }^{1, *}$, Minjun Seo, PhD. ${ }^{1}$, Michelle Khine, PhD. ${ }^{2}$, Josep Gumà Padró, MD. ${ }^{3}$, \\ Antonio Pardo Martínez, PhD. ${ }^{4}$, and Fadi Kurdahi, PhD. ${ }^{1}$
}

${ }^{1}$ University of California Irvine (UCI), Center for Embedded Cyber-physical Systems (CEPS), Irvine, 92697, USA

${ }^{2}$ University of California Irvine (UCI), Department of Biomedical Engineering, Irvine, 92697, USA

${ }^{3}$ Rovira i Virgili University (URV), South Catalonia Institute of Oncology (IISPV), Sant Joan University Hospital

(HUSJ), Reus, 43204, Spain

${ }^{4}$ Department of Electronics and Biomedical Engineering, Universitat de Barcelona (UB), 08028 Barcelona, Spain

*jgirbene@uci.edu

\begin{abstract}
A rising number of authors are drawing evidence to the diagnostic capacity of specific volatile organic compounds (VOCs) resulting from some body fluids. While cancer incidence in society is on the rise, it becomes clear that the analysis of these VOCs can yield new strategies to mitigate advanced cancer incidence rates. This paper presents the methodology implemented to test whether a device consisting of an electronic nose inspired after a dog's olfactory system and olfactory neurons is significantly informative to detect breast cancer (BC). To test this device, 90 human urine samples were collected from control subjects and BC patients at a hospital. To test this system, an artificial intelligence-based classification algorithm was developed. The algorithm was firstly trained and tested with data resulting from gas chromatography-mass spectrometry (GC-MS) urine readings, leading to a classification rate of $92.31 \%$, sensitivity of $100.00 \%$, and specificity of $85.71 \%(\mathrm{~N}=90)$. Secondly, the same algorithm was trained and tested with data obtained with our eNose prototype hardware, and class prediction was achieved with a classification rate of $75 \%$, sensitivity of $100 \%$, and specificity of $50 \%$.
\end{abstract}

\section{Introduction}

The US female population is 165.92 million women. The US Preventive Services Task Force (USPSTF) recommends biennial screening mammography for women aged 50-74 and recommends against screening younger and older women due to current evidence being insufficient to prove its benefits. ${ }^{1}$ This reduces BC mortality by $15 \% .^{2}$ According to the CDC, only $66 \%$ of women over 40 years of age do attend BC screenings annually or biannually. ${ }^{3}$ This can cause over $1 / 3$ of BC diagnoses to be detected too late. The main reason why women skip their mammogram appointment is pain. ${ }^{4}$ In the US, 451,936 BCs are detected yearly, ${ }^{5} 150,000$ of which are detected late-stage because of mammogram absenteeism or inefficiency of current methods. In fact, the impact of implementing a mammogram-based screening on breast cancer mortality has been under discussion for a very long time. A review by Moss et al. suggests that although most statistical studies report a decrease in breast cancer mortality over the years, such decrease can also be observed before the implementation of screening and in age ranges excluded from screening. ${ }^{6}$

If $\mathrm{BC}$ is detected in an early stage, its metastasis risk is minimal as well as the likelihood of a subsequent metastasis, patient suffering and death. If detected in a later stage, however, patients will require treatment and potentially a mastectomy, which has a notorious impact on women's mental health. These interventions have an approximate cost of $\$ 25,000$ per patient. Considering that $80 \%$ of the US population is covered by insurance, health insurances could save over $\$ 3$ billion yearly if all BCs detected in their patients were detected in an early stage. Additionally, apart from the mastectomy, if cancer progresses and the patient lives with metastasis, the cost of her treatment (monoclonal antibodies, sometimes conjugated with chemotherapy, cyclines inhibitors, hormone therapy, palliative radiotherapy...) is notorious. Blumen et al. report yearly costs per patient of up to $\$ 82,121$ for BCs detected in stage I/II as opposed to $\$ 129,387-\$ 134,682$ if detected in stage III/IV, mainly due to chemotherapy. ${ }^{7}$

$\mathrm{BC}$ is the leading cause of death by cancer in women under 40 years of age, especially among African-American women. They present a denser mammary tissue than post-menopause women, thus decreasing mammogram's sensitivity and specificity in small tumors. In addition to that, their tissue is more sensitive to the mammogram's radiation dose. ${ }^{8}$ Furthermore, in 2017, the World Health Organization (WHO) published the "WHO Position paper on mammography screening." 9,10 stating an urgent need for a new radiation-free and sensitive BC screening solution. Although its dose is not substantial enough to be considered 
harmful, biennially exposure to mammography could increase BC risk. ${ }^{11}, 12$ This observation, shared by several authors, started a debate on the worthiness of mammogram-based $\mathrm{BC}$ screenings. ${ }^{13}$ In other words, although mammography is currently the best solution to reduce $\mathrm{BC}$ mortality, there is still room for improvement.

\begin{tabular}{|c|c|c|c|c|c|}
\hline \multirow[b]{2}{*}{ Sensor type } & \multicolumn{5}{|c|}{ Published eNose approaches } \\
\hline & $\begin{array}{c}\text { Metal oxide } \\
\text { semiconductors }\end{array}$ & Gold nanoparticles & Quartz microbalance & Colorimetry & Cyranose 320 \\
\hline References & [41-43] & {$[36,37]$} & {$[38,39]$} & [44] & {$[45,46]$} \\
\hline $\begin{array}{l}\text { Electronic } \\
\text { fundament }\end{array}$ & $\begin{array}{l}2 \text { metal oxide } \\
\text { semiconductors and an } \\
\text { array of } 10 \text { metal oxide } \\
\text { semiconductor } \\
\text { field-effect transistor } \\
\text { sensors. }\end{array}$ & $\begin{array}{l}14 \text { GNP electrodes with } \\
\text { changing resistance when } \\
\text { in contact with VOCs. }\end{array}$ & $\begin{array}{l}\text { 8-sensor array of } \\
\text { oscillating quartz crystals } \\
\text { coated with varied } \\
\text { metalloporphyrins that } \\
\text { adsorb VOCs. }\end{array}$ & $\begin{array}{l}36 \text { spots impregnated } \\
\text { with chemically sensitive } \\
\text { compounds, forming an } \\
\text { array on a disposable } \\
\text { cartridge. }\end{array}$ & $\begin{array}{l}32 \text { built-in carbonblack } \\
\text { polymer composite } \\
\text { chemoresistors array and } \\
\text { a processor. }\end{array}$ \\
\hline $\begin{array}{l}\text { Physical } \\
\text { principle }\end{array}$ & $\begin{array}{l}\text { VOC-dependant } \\
\text { resistance that outputs a } \\
\text { voltage decay } \\
\text { proportional to the sensed } \\
\text { combination of VOCs. }\end{array}$ & $\begin{array}{l}\text { Change in physical } \\
\text { properties depending on } \\
\text { their size and shape } \\
\text { (altered by contact with } \\
\text { VOCs). }\end{array}$ & $\begin{array}{l}\text { Adsorption of VOCs by a } \\
\text { thin quartz crystal area } \\
\text { results in a variation of } \\
\text { its mass, which induces a } \\
\text { variation of the } \\
\text { oscillation frequency. }\end{array}$ & $\begin{array}{l}\text { Adsorption of VOCs to } \\
\text { the dots of } \\
\text { metalloporphyrins causes } \\
\text { them to change in color. } \\
\text { Change is quantified and } \\
\text { denotes VOC } \\
\text { composition. }\end{array}$ & $\begin{array}{c}\text { Sensor swells upon } \\
\text { VOCs exposure causing } \\
\text { an increase in electrical } \\
\text { resistance. }\end{array}$ \\
\hline
\end{tabular}

\begin{tabular}{c|ccccc}
\cline { 2 - 4 } & Cancer type, \\
sample & $\begin{array}{c}\text { Bladder cancer, } \\
\text { headspace urine sample. } \\
\text { Prostate cancer, cell } \\
\text { medium }\end{array}$ & $\begin{array}{c}\text { Breast, lung, colorectal, } \\
\text { prostate cancer, breath } \\
\text { samples }\end{array}$ & $\begin{array}{c}\text { Lung cancer, breath } \\
\text { samples (headspace) }\end{array}$ & $\begin{array}{c}\text { Lung cancer, breath } \\
\text { samples }\end{array}$ & $\begin{array}{c}\text { Lung cancer, pleural } \\
\text { cancer; breath samples } \\
\text { (solid-phase) }\end{array}$ \\
\end{tabular}

Specifications \begin{tabular}{c|ccccc|}
$\begin{array}{c}\text { Convenient due to its } \\
\text { small size, no need for } \\
\text { high voltage, and high } \\
\text { sensitivity (i.e. no } \\
\text { complex pre-processing } \\
\text { needed). }\end{array}$ & $\begin{array}{c}\text { Performs irrespective of } \\
\text { age, gender... Separates } \\
\text { early from the advanced } \\
\text { stage. }\end{array}$ & $\begin{array}{c}\text { Good sensitivity for } \\
\text { alcohols and ketones and } \\
\text { for breath affections } \\
\text { apart from lung cancer. }\end{array}$ & $\begin{array}{c}\text { Results are not affected } \\
\text { by the subjects' } \\
\text { demography, smoking }\end{array}$ & $\begin{array}{c}\text { Might as well apply to } \\
\text { the detection of bacterial } \\
\text { pathogens, blood glucose }\end{array}$ \\
histor stage of cancer. & level, bronchogenesis.... \\
\hline
\end{tabular}

\begin{tabular}{|c|c|c|c|c|c|}
\hline Performance & $\begin{array}{l}70 \% \text { accuracy, } n=89 \\
94 \% \text { accuracy, } n=27\end{array}$ & $88 \%$ accuracy, $n=72$ & $\begin{array}{c}94 \% \text { accuracy, } \\
n=44 ; \\
85 \% \text { sensitivity, } n=98\end{array}$ & $73.3 \%$ specificity, $n=143$ & $\begin{array}{c}71.4 \% \text { sensitivity, } \mathrm{n}=76 \\
90 \% \text { accuracy, } \mathrm{n}=30\end{array}$ \\
\hline
\end{tabular}

Figure 1. Summary of published eNose approaches to portable low-cost screening solutions: Metal oxide semiconductors, gold nanoparticles, quartz microbalance, colorimetry and the Cyranose 320.

\section{Current methods for breast cancer screening}

Most healthcare systems base their BC screening programs on image-based technologies, i.e. mammography, ultrasonography and magnetic resonance imaging (MRI). The most common reason why women seek medical advice related to $\mathrm{BC}$ is having detected a mass in their breasts. However, $90 \%$ of these will be found to not be cancer but other benign lesions such as fibroadenoma. ${ }^{14}$ Actually, image-based techniques typically fail to correctly identify tumors in women with fibrocystic breasts (breast tissue with healthy lumps) because of highly dense breast tissue. ${ }^{15}$ Around 53-60\% of women worldwide have this condition. ${ }^{16}$ This fact does not only lead to numerous false positives but also increases the recommended screening frequency (and thus the potentially cancerogenic small radiation dose).

When the tumor is small or the mammary tissue is dense, MRI is typically the preferred approach, since it is highly specific and sensitive in addition to being a radiation-free technique. For this reason, BRCA1 or BRCA2-positive women (who present a high risk of developing BC) are screened from a younger age using MRI. Women with fibrocystic mastopathy (whose breasts have difficult tumor visualization) and patients whose mammogram results are inconclusive are typically screened with MRI as well. ${ }^{17}$ However, screening $1 \mathrm{M}$ women costs $\$ 640 \mathrm{M}$ and $\$ 216 \mathrm{M}$ if done with MRI and mammography respectively. ${ }^{18}$ There is therefore a tendency of enhancing mammography by processing the resulting image with an AI-based classification algorithm. 
However, even if this solution can potentially greatly increase the mammogram's sensitivity, it does not solve another problem: this technique is painful to some women and thus causes screening absenteeism.

\section{Scientific principle}

The medical community has long accepted the fact that BC produces metabolic changes in human physiology. ${ }^{19-22}$ More specifically, Vignoli et al emphasise on a "BC metabolomic signature in breast tissue, blood, serum/plasma and urine", detected through Nuclear Magnetic Resonance spectroscopy (NMR). ${ }^{23}$ However, traditional observational approaches and current urinalysis to detect biomarkers in urine have been proved not to be informative enough. Since the ' 90 s, an increasing number of publications have been seeking the definitive set of BC biomarkers. This would lead to an early-stage metabolites-based cancer test. Nonetheless, there does not exist a consensus among authors yet: A metanalysis by Dent et al. ${ }^{24}$ reviews the findings from several authors from 2003 up to 2012 and concludes that the "VOC fingerprint" differs significantly among publications. In conclusion, the key to successfully identifying cancer is focussing on the proportion between VOCs rather than on VOCs themselves.

In this project, a different approach to the challenge was followed - based on engineering rather than observational medicine: Multidimensional data obtained from the sample can then be analyzed by statistical algorithms. Like the human nose, the implemented software will respond in concert to a given set of odors -a pattern or "smellprint"- which is analyzed, compared with stored patterns, and recognized. The presented device is based on the principle that BC causes certain inflammatory processes, resulting in specific circulating metabolites. These subsequently interact with the excretory system, which translates into the urine of $\mathrm{BC}$ patients containing the decomposition products of these metabolites.

Finally, one has to take into account that urine's metabolome is highly dependant on diet, environment and lifestyle, and consequently has more daily variability than serum or plasma. ${ }^{23}$ The metabolic fingerprint of BC in urine has been less studied than that of serum, plasma and breast tissue, but Vignoli et al report the findings from 4 different studies that coincide on the following: $\mathrm{BC}$ is associated with downregulation of acetate, alanine, creatinine, dimethylamine, glutamine, glycine, guanidoacetate, hippurate, isoleucine, lactate, leucine, succinate, taurine, threonine, trimethylamine n-oxide and valine. ${ }^{23}$ Finally, this metanalysis also highlights the potential of this becoming a new tool for early-stage BC screening.

\section{State of the art. Electronic noses}

Even though there do exist some publications on odor-based cancer screenings -i.e. electronic noses or eNoses-, the tech transference rate is extremely low. The first eNose, proposed by Persaud and Dodd ${ }^{25}$ in Nature in 1982, was designed to loosely mimic the human olfactory pathway. An eNose is based on a sensor array that responds differently according to specific VOCs. In fact, the sensor's response is not unequivocally correlated to the concentration of a single compound but rather a combination of the chemical information of a sample. A variety of electronic noses described in the bibliography are subject to urine or exhaled alveolar breath and commonly apply to a wide variety of diseases, but none of them are designed specifically for BC. In 2014, Asimakopoulos et al. ${ }^{26}$ set the race towards an eNose approach to cancer screening with the first study on an eNose-based prostate cancer identification in a non-invasive, quick and easy manner, achieving a specificity of $93 \%$ ( $\mathrm{n}=41)$.

Furthermore, Guerrero-Flores et al. ${ }^{27}$ describes a cervix cancer screening in which a dog detects cancer cells in blood by smelling it. Buszewski et al. ${ }^{28}$ also presents a VOC-based screening from breath through canine smell and Blatt et al. ${ }^{29}$ describes the same screening carried out by an "artificial nose" that analyzes VOCs and processes them with a classification algorithm that implements statistical analysis such as Principal Components Analysis, Linear Discriminant analysis and other biostatistics techniques. A considerable amount of publications of this kind focus on lung, prostate and BC. As far as the latter is concerned, Phillips et al. ${ }^{30}$, describe a BC screening using an electronic device that analyzes the patient's breath.

Burton et al. ${ }^{32}$ and Guo et al. ${ }^{33}$ among other authors comment on the same type of screening from urine samples. In this case, not only does it detect the presence of cancer but also the stage: I and II (early) versus III and IV (advanced). However, in these studies, the dogs may have responded to odors associated with cancer, such as inflammation or metabolic products, rather than specifically to cancer itself. ${ }^{34}$ Hence, the future of volatilome-based screening should not seek a cancer odor but rather a specific VOC pattern for each cancer type, which has already been proven feasible with BC among others. In conclusion, the sensitivity of this technique is sufficient to assist doctors in identifying cancer in addition to the most optimal treatment. ${ }^{35}$

\section{Technologies for artificial odoring}

Some electronic noses have already reached the industry: vReCIVA® Breath Sampler by Owlstone Medical is a VOC sensing instrument that analyzes breath to detect lung cancer. Similar to that, Aeonose is a certified medical device intended for disease screening using exhaled-breath. It can screen colorectal cancer with a sensitivity $95 \%$ and specificity $64 \%$ and advanced adenomas with sensitivity and specificity $79 \%$ and $59 \%$ respectively $(\mathrm{N}=511)^{36}$. On the other hand, NASA has also developed an eNose, a smartphone-based device to monitor air quality inside spacecraft. On top of that, they have recently modified it to screen people for COVID-19 in a low-cost and efficient manner. Heracles by Alpha MOS is an electronic nose based on ultra-fast gas chromatography that is able to classify wines ${ }^{37}$, olive oil ${ }^{38}$ and other substances. 
Although being the most successful and well-performing approach according to most authors, ${ }^{24}$ metal-oxide sensors are not the only low-cost and portable solution for artificial odoring. An eNose based on a nanosensor array with gold nanoparticles $(\mathrm{GNP})^{39,40}$ or a quartz microbalance ${ }^{41,42}$ are different types of sensors that have proven high sensitivity and specificity applied to $\mathrm{BC}$ and lung diseases respectively. Peng et al. ${ }^{39}$ present a tailor-made array of cross-reactive nanosensors based on organically functionalized gold nanoparticles and the GC-MC technique (GC-MS). This nanosensor array could distinguish between the breath patterns of different cancers in the same statistical analysis, irrespective of age, gender, lifestyle, and other confounding factors $(n=96)$. Fig. 1 summarises various eNose descriptions found in bibliography.

\section{Results}

\section{Software considerations. Classic biostatistics approach}

As depicted in Fig. 10, the first approach consisted of building a first model for sample classification based on classic biostatistics. Before classifying the data, it has been pre-processed to ensure that the classification will, later on, rely on the characteristic of cancer instead of on irrelevant artifacts. Pre-processing steps are described in Fig. 2. Later on, a Principal Components Analysis (PCA) was performed, and it was used to prove the hypothesis mentioned below:

\section{Urine samples from breast cancer patients are significantly different from control samples.}

The PCA was firstly conducted using GC-MS data. A visual inspection of this classification is displayed in Fig. 3 A and B. Once observed that it was possible to discriminate control samples from BC samples, the same procedure was repeated by analyzing the same data with the eNose prototype. This time, the classification algorithm was simplified so that it could run on the edge - e.g., in the microcontroller inside the eNose. It was thus a simplified PCA, consisting of only 2 Principal Components (PCs). The projection of urine samples against PC1 and PC2 is shown in Fig. $3 \mathrm{C}$ and D.

Hence, the scientific principle for BC eNose was proved. Below we compare the smellprint, obtained from two data sources: GC-MS-acquired data (Fig. 3 A and B), versus the eNose-captured signal (Fig. 3 C and D).

\section{Software considerations. A machine learning approach}

Once the null hypothesis has been tested, an AI-based algorithm has been implemented to assert the classification capacity of a convolutional neural network (from now on, CNN). This neural network consisted of four convolutional filters of size 32, 64, 128 and linear activation. The output layer of the neural network presented two cells (two outputs) with a softmax activation function. Fig. 4 is a sketch of how the model structure was designed. As indicated, dropout was implemented to avoid model overfitting. In our first approach to the CNN (ConvNet) model, GC-MS data was used: 90 urine samples from control and BC subjects. This model was trained with 50 epochs of batch size 10. Out of 90 samples, 65 (72.3\%) samples were used for training, 12 (13.3\%) for testing, and $13(14.4 \%)$ for cross-validation. The achieved training accuracy was $98.20 \%$, and the training loss was $7.70 \%$. Later on, the validation accuracy was $\mathbf{9 2 . 3 1 \%}$. As displayed in Table 1, the model has a sensitivity of $\mathbf{1 0 0 \%}$ and a specificity of $\mathbf{8 5 . 7 1 \%}$. For more detail, we present a confusion matrix in Table 1.

\begin{tabular}{|c|c|c|}
\hline & $(+)$ predicted & $(-)$ predicted \\
\hline (+) ground truth & 6 & 0 \\
\hline (-) ground truth & 1 & 6 \\
\hline
\end{tabular}

Table 1. Confusion matrix of the CNN model using GC-MS data. Out of 90 samples, 65 (72.3\%) samples were used for training, $12(13.3 \%)$ for testing and $13(14.4 \%)$ for validation.

Similar to the previous section, the model was subsequently trained and tested with eNose-obtained data. Out of 44 samples, $36(81.8 \%)$ samples were used for training, $4(9.1 \%)$ for testing, and $4(9.1 \%)$ for validation. The structure of the model was the same as presented in Fig. 4. In this section we provide a confusion matrix with the obtained results, see Table 2. The achieved training accuracy was $93.3 \%$ and the training loss was $14.72 \%$. Validation accuracy was $75.00 \%$. As displayed in Table 2 , the model has a sensitivity of $100.00 \%$ and specificity of $50.00 \%$.

\begin{tabular}{|c|c|c|}
\hline & (+) predicted & $(-)$ predicted \\
\hline (+) ground truth & 2 & 0 \\
\hline (-) ground truth & 1 & 1 \\
\hline
\end{tabular}

Table 2. Confusion matrix of the $\mathrm{CNN}$ model using data acquired by the eNose prototype. 36 (81.8\%) samples were used for training, $4(9.1 \%)$ for testing and $4(9.1 \%)$ for validation. 
Interpolation along time and $\mathbf{m} / \mathbf{z}$ axis. Each $2 \mathrm{D}$ sample reading informs of the intensity at which VOCs of each size (mass/charge, i.e. $\mathrm{m} / \mathrm{z}$ ) are found in urine per instant of time. - Because all samples have been analyzed the same number of times but not at the same specific times, time interpolation is needed. interpolate $t$ ( ) function takes as input the evolution of intensity along time and uses this data to predict the behavior of the function S at the desired times. The same logic is used to interpolate over $\mathrm{m} / \mathrm{z}$ with interpolate $m z()$. Mass spectra have been predicted as if sampled 1000 equispaced times within $30-300$ $\mathrm{m} / \mathrm{z}$ and 3000 times between $2-20 \mathrm{~min}$

Chromatogram design. Thanks to interpolation, data can now be integrated along the N $\mathrm{m} / \mathrm{z}$ axis and therefore convert a 3D signal (intensity v.s. time v.s. $\mathrm{m} / \mathrm{z}$ ) into a $2 \mathrm{D}$ signal: The evolution of the intensity of all masses along time. From this point onwards, a 5 subject is described by a single vector, the total ion chromatogram (TIC). The resulting TIC is the blue signal presented below.

$m$ Baseline removal. GC-MS records the signal together with added noise that increases over time. This baseline low-frequency noise and drift modifications have been removed 든 utilizing baseline removal(), which does not subtract from the sample when intensity is increase is not due to noise but an actual chromatogram peak.
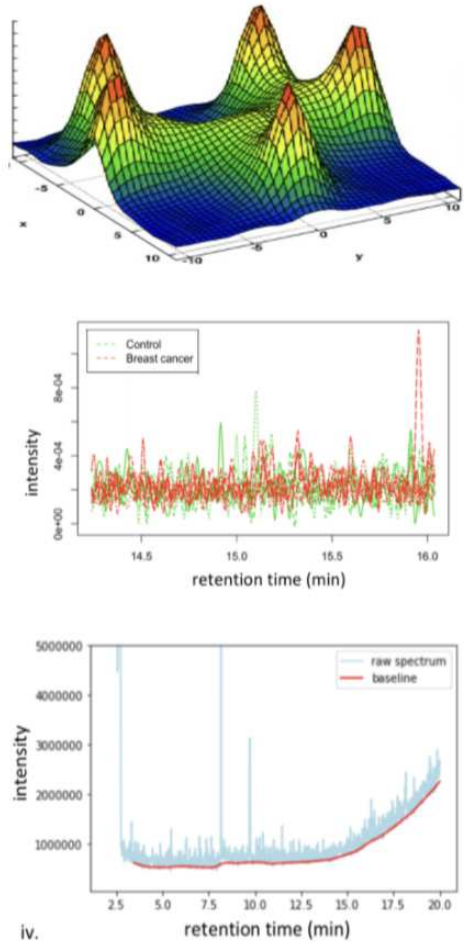

Savitzky-Golay filter. $S G($ ) takes each point of the chromatogram together with their 30 $\nabla$ surrounding neighbors and estimates the parameters of the 11-order polynomial that best fits this data. This polynomial is then used to set the intensity of the central point to the value the polynomial takes at this place and reduce additive high-frequency noise. An in optimal SG filter removes high-frequency contributions to the signal caused by noise but ignores low frequencies.

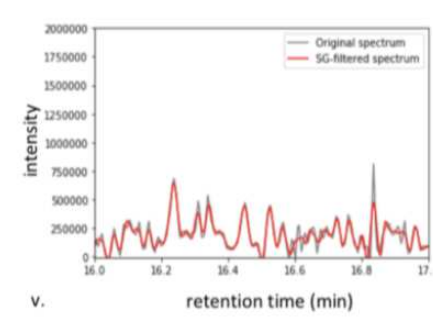

in Normalization. It prepares the feature vector for the next pattern analysis steps and ensures subjects are comparable among them $\hat{7}$ and classifications do not rely on water consumption per day or diuresis rate i.e. classification is not quantitative but qualitative However, the area under the curve of large peaks contributes largely to that of the TIC, leading to over-classified samples. By not is considering the area under peaks higher than 106, the enhanced normalize avoidPeak() does not over-normalize.

3-fold sample averaging. To seek repeatability and disregard features related to GC-MS

performance, each sample has been analyzed three times, pre-processed, and then averaged. Samples are 4600-dimensional vectors with an intensity value per instant of time $(2-20 \mathrm{~min})$

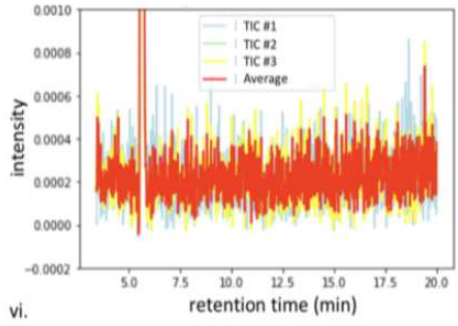

Figure 2. Summary of the pre-processing steps from GC-MS raw data reading up to total ion chromatogram (TIC) design. Sample 151 (belonging to a breast cancer subject) has been used to illustrate those steps 

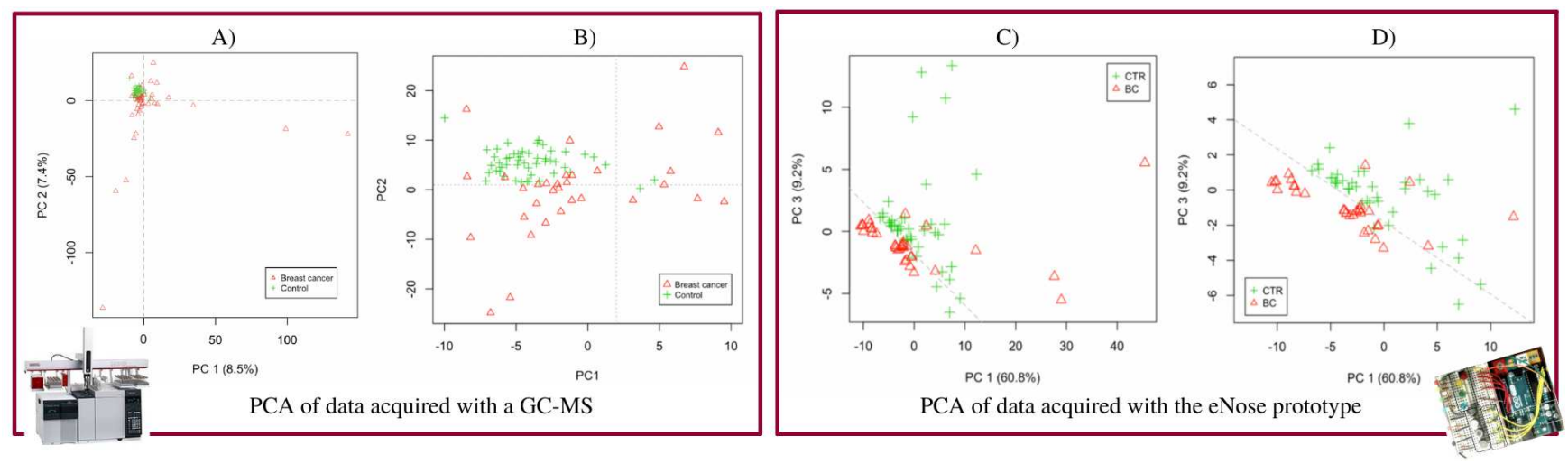

Figure 3. A) PCA performed on data obtained by analyzing control (green) and BC (red) human urine with a GC-MS. B) Figure on the right is a zoom of the figure on the left. C) PCA performed on data obtained by analyzing control (green) and BC (red) human urine with the eNose prototype. D) Figure on the right is a zoom of the figure on the left.

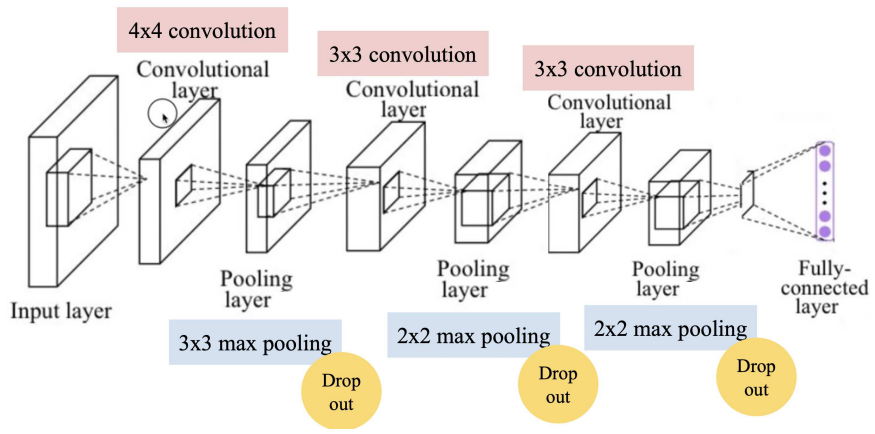

Figure 4. Convolutional neural network used for sample classification, consisting of convolutional filters of size 32, 64, 128 and linear activation. The output layer of the neural network presents two cells (two outputs) with a softmax activation function.

\section{Accuracy, sensitivity and specificity}

Upon collection, urine samples were first analyzed using a gas chromatographer-mass spectrometer (GC-MS), which provided an insight on the intensity in which every type of molecule was found at every retention time. In other words, the output consisted of a report for every retention time, which indicated the number of molecules found at that given time for every molecular mass, relative to their ionized charge. Fig. 10 shows the shape of the GC-MS data. Later on, this data was processed by a PCA and CNN, which will be detailed as follows. Secondly, urine samples were also analyzed utilizing the eNose prototype that we developed. Please refer to section Method for a detailed description of the prototype. The output of this second analysis was a 2D matrix containing the response of the 4 sensors at every instant of time. As one can observe in Fig. 10, this data was classified by a PCA and a CNN as well. Table 3 displays the accuracy of the two models that were described before: the biostatistics model and the AI-based model respectively.

\begin{tabular}{|c|c|ccc|c|}
\hline & Technique & Accuracy & Sensitivity & Specificity & Sample size \\
\hline \hline \multirow{2}{*}{ PCA } & GC-MS data & $77.11 \%$ & $75.05 \%$ & $68.33 \%$ & $\mathrm{~N}=90$ \\
& eNose data & $58.30 \%$ & $75.00 \%$ & $45.00 \%$ & $\mathrm{~N}=44$ \\
\hline \hline \multirow{2}{*}{ cNN } & GC-MS data & $92.31 \%$ & $100.00 \%$ & $85.71 \%$ & $\mathrm{~N}=90$ \\
& eNose data & $75.00 \%$ & $100.00 \%$ & $50.00 \%$ & $\mathrm{~N}=44$ \\
\hline \hline Mammography & - & $>91.00 \% 43$ & $86.90 \% \%^{23}$ & $74.00-98.00 \%$ & - \\
\hline
\end{tabular}

Table 3. Sample classification using biostatistics (PCA) and machine learning (cNN) vs reported performance of mammography

As one can observe, the introduction of neural networks plays a critical role in enhancing the classification capability of 
the system. Additionally, it should also be noted that the model classification rate is highly dependent on the sample space size. Therefore we consider it necessary to conduct further studies to keep training the algorithm and achieve a sensitivity and specificity that are acceptable for an oncology screening.

\section{Discussion}

As these results denote, machine learning -in this case, CNN- causes a significant impact on sample classification: In the presented case, the accuracy of the model rises from $58.30 \%$ to $75.00 \%$ (when using eNose data). Additionally, it should be noted that the eNose data set has a smaller dimensionality. Additionally, because this technology is intended for screening, special attention should be placed on sensitivity rather than specificity. In our case, if we compare our AI-powered model to mammography, our sensitivity is notabaly higher (100\%). Mammography has a sensitivity of $86.9 \%$ and its performance is highly dependant on age and tissue density ${ }^{23}$.On the other hand, our highest specificity value is achieved when implementing CNN with GC-MS data. However, the authors believe that better specificity might be obtained with eNose data if enough data were available.

The experiments carried out in this paper indicate that the implementation of AI in the medical field can yield new approaches and discoveries that classic biostatistics could not reach. This paper also suggests the feasibility of a future potential medical device for in-home and non-irradiating BC screening. As denoted by the size of the training set, additional studies are yet to be conducted to better identify the classification capacity of the technology. The results presented above were achieved with a samples size of 90 patients. Therefore, the authors believe it is necessary to carry out a new round of patient studies, targeting an order on the magnitude of 300-500 patients.

\section{Theoretical fundament for 8-oxodG presence in breast cancer urine samples}

When it comes to $\mathrm{BC}$, publications on its specific biomarkers are currently rare yet consistent. Some articles point out that an increased presence in the urine of the volatile 8-oxo-7,8-dihydro-2'-deoxyguanosine, shown in Fig. 5, (from now on, 8 -oxodG) denotes cancer. Guo et al. ${ }^{33}$ found a significant increase of 8 -oxodG in patients with early-stage BC (p<0.001) by ultraperformance liquid chromatography-electrospray ionization tandem mass spectrometry combined with a solid-phase microextraction $(n=184)$.

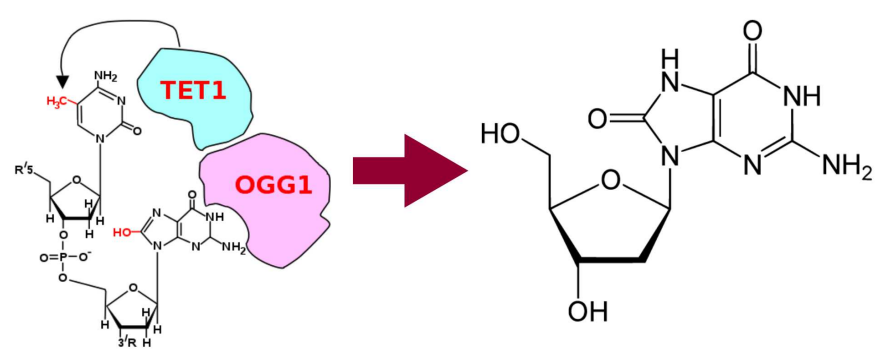

Figure 5. Formation of 8-oxodG out of guanine from a DNA sequence with intervention of TET1 and OGG1. 8-oxodG is a sensitive marker of DNA damage due to hydroxyl radical attack at the C8 of guanine. This damage is mutagenic and promotes cancer.

According to Guo et al., reactive oxygen species (ROS) are produced by endogenous oxygen metabolism, as well as after exposure to ionizing radiation and chemical carcinogens. ${ }^{50}$ The enzyme TET1 catalyzes a reaction with a guanine. For this reaction to happen, the guanine needs to have been oxidized to 8-hydroxy-2'-deoxyguanosine (8-OHdG or its tautomer 8-oxodG) due to the presence of a ROS. This oxidation allows enzyme OGG1 to bind to 8-oxodG and thus recruits TET1, which oxidizes the molecule, see Fig. 5. Increased ROS can cause oxidative base modifications and thus lesions in DNA. Since guanine exhibits the lowest oxidation potential, it is more vulnerable to free a radical, leading to the formation of 8-oxodG, commonly conceived as a biomarker of oxidative damage to DNA and a mutagen that contributes to carcinogenesis. Those features imply that a urine-based screening for 8-oxodG would be non-invasive, the patient would not get irradiated and it would not result in false positives, with the consequent economic savings. ${ }^{27,33,51}$

\section{Theoretical fundament for benzoic acid absence in BC urine samples}

Benzaldehyde is the only downregulated component by BC. It is absorbed via the gastrointestinal tract, skin and lungs, then distributed -especially in the blood and kidneys-, and finally excreted very rapidly almost exclusively via urine. During the process, benzaldehyde gets oxidized to benzoic acid. ${ }^{52}$ Hence, if Lavra et al. ${ }^{35}$ reported a lack of benzaldehyde in BC cells mediums, a lack of benzoic acid should be expected in BC patients' urine. In fact, the PubChem database ${ }^{53}$ confirms benzoic 
acid presence in control urine within the range 350-630 nmol/mmol creatinine. Therefore, some of the sensors inside the analysis chamber have been specifically selected to react to benzenes and benzoic acid.

\section{Theoretical fundament for 2-nonanone presence in breast cancer urine samples}

Lavra et al. ${ }^{35}$ suggests 2-nonanone, 4-methil-2-heptanone, isobutyric acid allyl ester, 1,3-dis-ter-butylbenzene and benzaldehyde among others as cancer biomarkers encountered directly in BC cell mediums. As depicted in Table 4, such components exhibit a significant difference in concentration between control cell medium and primary tumor cell medium (BT474) with respective p-values of $6.5 \cdot 10-21,5.8 \cdot 10-11,3.6 \cdot 10-10,1.8 \cdot 10-9$ and 0.013 . Table 1 shows a summary of the different VOCs found in human physiology according to many authors.

\begin{tabular}{|c|c|c|c|}
\hline & Bening breast tissue (MCF10A) & Cancerous breast tissue (BT474) & p-value \\
\hline 2-nonanone & Very low & Very high & $6.5 \cdot 10-2$ \\
\hline 4-methil-2-heptanone & Very low & Very high & $5.8 \cdot 10-11$ \\
\hline Isobutyric acid allyl ester & Very low & Very high & $3.6 \cdot 10-10$ \\
\hline 1,3-dis-ter- butylbenzene & Very low & Very high & $1.8 \cdot 10-9$ \\
\hline benzaldehyde & Very high & Very low & 0.013 \\
\hline
\end{tabular}

Table 4. Intensity of concentration of various $\mathrm{BC}$ biomarkers encountered in cell media: control, benign breast tissue (MCF10A), and cancerous breast tissue (BT474).

\begin{tabular}{|c|c|c|c|}
\hline \multicolumn{4}{|c|}{ Breast cancer volatile biomarkers } \\
\hline Urine & \multicolumn{3}{|c|}{ Cell medium } \\
\hline Guo et al. [19] & Lavra et al. [35] & Silva et al. [22] & Huang et al. [20] \\
\hline $\begin{array}{l}\text { 8-oxo-7,8-dihydro-2'- } \\
\text { deoxyguanosine }\end{array}$ & $\begin{array}{l}\text { Benzaldehyde } \\
\text { (downregulated) }\end{array}$ & $\begin{array}{l}\text { 2-Pentanone } \\
\text { 2-heptanone }\end{array}$ & $\begin{array}{l}\text { 1,4-dimethyl-benzene } \\
\text { (redox product) }\end{array}$ \\
\hline (8-oxodG) & $\begin{array}{l}\text { Prop-2-enyl 2-methyl } \\
\text { propanoate }\end{array}$ & $\begin{array}{l}\text { 3-methyl-3-buten-1-ol } \\
\text { ethyl acetate }\end{array}$ & $\begin{array}{l}\text { Cyclohaxanol } \\
\text { 2-ethylhexan-1-ol }\end{array}$ \\
\hline IUPAC name: & 2-nonanone & ethyl propanoate & 2,4-dimethyl-benzaldehyde \\
\hline $\begin{array}{l}\text { 2-amino-9-[(2R,4S,5R)-4 } \\
\text {-hydroxy-5-(hydroxymet } \\
\text { hyl)oxolan-2-yl]-3,7- }\end{array}$ & $\begin{array}{l}\text { 4-methil-2-heptanone } \\
\text { 1,3-dis-ter-butylbenzene }\end{array}$ & 2-methyl butanoate & (highly upregulated) \\
\hline & \multicolumn{3}{|c|}{ Exhaled breath } \\
\hline Chemical formula: & Brooks et al. [48] & Phillips et al. [30] & Li et al. [31] \\
\hline $\mathrm{C}_{10} \mathrm{H}_{13} \mathrm{~N}_{5} \mathrm{O}_{5}$ & 5-nonane & Alkanes derivatives & Hexanal \\
\hline Structure: & $\begin{array}{l}\text { 6-ethyl-3-octyl ester } \\
\text { 2-trifluoromethyl } \\
\text { benzoic acid } \\
\text { Nonane } \\
\text { propane, 2-methyl } \\
\text { nonadecane, 3-methyl }\end{array}$ & $\begin{array}{l}\text { Benzene derivatives } \\
\text { Pentane }\end{array}$ & $\begin{array}{l}\text { Heptanal } \\
\text { Octanal } \\
\text { Nonanal }\end{array}$ \\
\hline
\end{tabular}

Figure 6. Summary of some VOCs encountered in urine, cell medium and expired breath, according to Guo et al., Lavra et al., Silva et al., Huang et al., Brooks et al., Phillips et al. and Li et al. Those VOCs whose evidence was found to be more consistent and their derivatives have been highlighted.

\section{Theoretical fundament for acetone and 2-butanone in control urine samples}

In the last years, the urge to deeply characterize the VOCs present in BC patients' urine has led to the need for a deeper understanding of control urine volatiles as well. Because VOCs present in urine are numerous, any analysis searching for a specific VOC will result in an extremely noisy and superposed signal with several VOC signals to be filtered out. It is long well-known that control urine consists on a mixture of water $(91-96 \%)$, urea $(9.3 \mathrm{~g} / \mathrm{dL})$, creatinine $(0.670 \mathrm{~g} / \mathrm{L})$, sodium $(1.17$ $\mathrm{g} / \mathrm{L})$, potassium $(0.750 \mathrm{~g} / \mathrm{L})$, chloride $(1.87 \mathrm{~g} / \mathrm{L})$ and several VOCs ${ }^{54}$ Regarding the latter, it is most authors' belief that acetone and 2-butanone are the two predominant control VOCs in urine. Mochalski et al. ${ }^{55}$ performed a selective reagent ionization 
time of flight mass spectrometry and gas chromatography and headspace solid-phase microextraction to determine VOCs in human urine $(n=19)$. A total of 16 VOCs exhibiting high incidence rates were quantified in urine. Amongst them, there were ten ketones, three volatile sulfur compounds and three heterocyclic compounds (furan, 2-methylfuran, 3-methylfuran). According to this study, acetone (C3H6O-NO+) has a parent ion $\mathrm{m} / \mathrm{z}$ of 88.04 , and when analyzed with a GC-MS, it appears at retention time $\mathrm{Rt}=16.08 \mathrm{~min}$, very early, at an intensity of 3.0-52,000 nmol/1, i.e. extremely variable among samples. 2-butanone $(\mathrm{C} 4 \mathrm{H} 8 \mathrm{O} \cdot \mathrm{NO}+)$ has a parent ion $\mathrm{m} / \mathrm{z}$ of 102.06 , and when analyzed with a GC-MS, it appears at retention time $\mathrm{Rt}=22.22 \mathrm{~min}$, at an intensity of 0.9-637 nmol/l, i.e. its peak is less intense than acetone's. Fig. 6 summarizes the main findings of VOCs in urine according to most authors. $* * *$

\section{Methods}

\section{Overall system design}

System design consisted of three steps: identifying the targeted VOC biomarkers; building a proof of concept to gather data; and building the final prototype based on previous observations.

\section{The sensing unit}

The proposed eNose is based on a metal oxide sensor array that scans the olfactory imprinting of the initial part of the urine stream. Metal oxide semiconductors are widely used when designing an eNose since they are easily available at the market, non-expensive and notably well-performing. As depicted in Fig. 7, their surface conductivity changes upon adsorption and subsequent reaction of gases with the already-adsorbed oxygen. Since this reaction is an oxidation or reduction one -in the case of $\mathrm{SnO}$ or $\mathrm{ZnO}$ sensors-, the concentration of electrons available for conduction gets altered and causes this material to be an optimal semiconductor. ${ }^{46}$

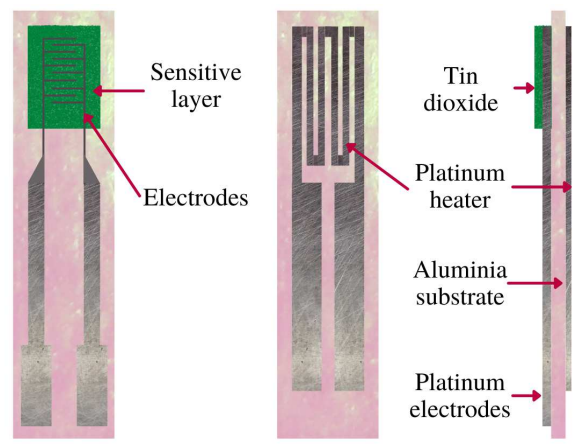

Figure 7. Structure of a metal oxide sensor. The sensitive layer on the top reacts with the presence of certain VOCs and thus sensors change in conductivity. The platinum heater placed on the bottom of the sensor consists of a dissipating resistance that outputs heat. By heating the sensor a higher VOC specificity is reached.

\section{Proof of concept}

This approach was built to prove whether the analysis of human urine could be performed in a portable and low-cost way. The proof of concept consisted of a breadboard with 4 VOC-specific sensors able to capture the smellprint of urine as well as a microprocessor to control the system. The data was analyzed on edge, and results were displayed through a color-coded LED-based user interface shown in Fig. 8. The four gas sensors on the breadboard are the key components of the electronic system thanks to the fact that the $\mathrm{SnO} 2$ layer (Fig. 7) absorbs the VOCs present in the sample and thus changes in conductivity. However, this phenomenon is only possible at a given temperature, which is why $5 \mathrm{~V}$ need to be continuously supplied to the sensor's heater plate, which keeps the sensitive layer warm. The heater is an $83 \Omega$ resistance working at $42 \mathrm{~mA}$. Finally, metal oxide sensors are poorly selective in ambient temperature with a high presence of water vapor, ${ }^{56}$ and thus reach optimal performance at $68 \mathrm{~F}, 65 \% \mathrm{HR}$. Arduino board also outputs voltage and ground to feed the electronic components of the system. In this case, the 5V and GND outputs were used (Fig. 8, orange and green wires). This is the prototype that was used to acquire the 44 urine smellprints mentioned in the Results section.

\section{eNose final prototype}

Based on the proof of concept mentioned above, this second prototype was aimed at better capturing the signal (i.e., to profile the smell of the sample more accurately) and allow for a more complex algorithm. This prototype, shown in Fig. 8, was not tested in a clinical setting. The final prototype, designed along with the dog's olfactory system and neurons, consists of a 


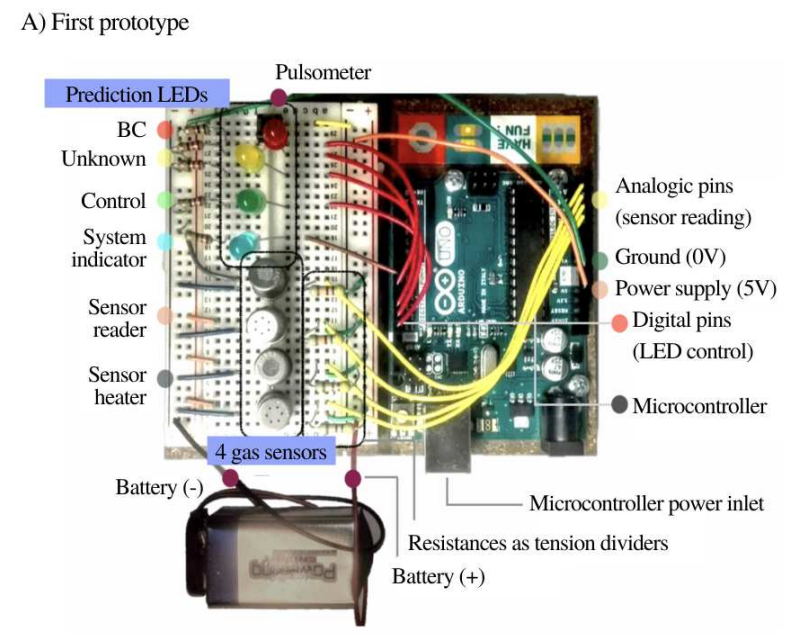

B) Next prototype

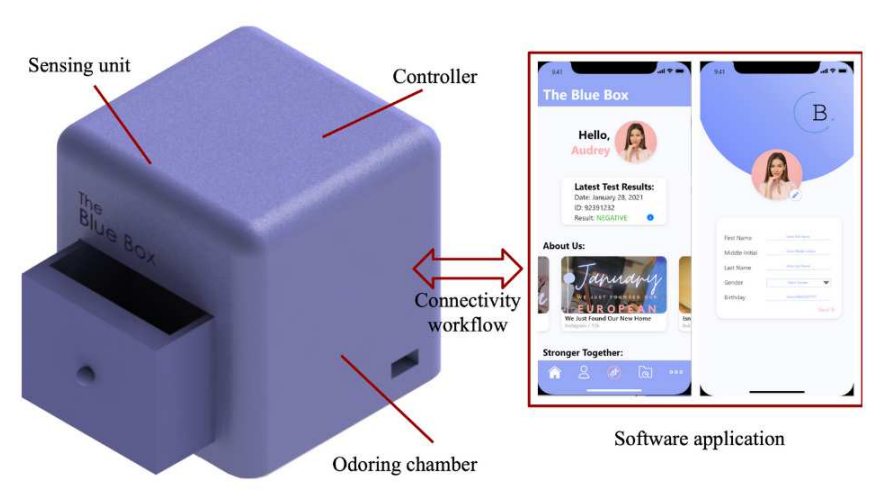

Figure 8. (A) Main electronic components that integrate the first eNose prototype. The 4 gas sensors "sniff" the sample and send a VOC-dependant 0-5V signal to the Arduino's analogic pins. Their combined signal is then reduced in dimensionality and undergoes sample classification. Depending on the prediction, output digital pins supply current to the corresponding LED, alerting of "possibly BC", "prediction no strong enough" (Blank) or "possibly control" (CTR). (B) Structure of the device and software application that we mentioned above. The 3D-printed structure contains all parts involved in the urine analysis. The software application is the interface to trigger the analysis and receive the results.

3D-printed inner structure where to allocate a urine sample that allowed an optimal collection of the urine sample, as shown in Fig. 8. The system hence consists of the following:

- Odoring chamber: a physical structure where to collect the smell of urine. The chamber consists of an irradiating wall that dissipates heat over time in a controlled fashion. By doing so, it is easier to assess which VOC is evaporated at every instant of time, depending on their boiling points.

- Sensing unit: array of sensors that acquire the smellprint

- Controller: microprocessor that gathers all data and executes the embedded code

- Connectivity workflow: to send the captured information via Bluetooth Low Energy to the software application and via the internet to the cloud-based server

- Cool \& clean system: creating an airflow through the device to keep the electronics from overheating and cleans the Sensing unit once the analysis is has finished

- Software application: a mobile application installed at the user's smartphone to collect the patient's demographic data, merge it with sensor data and forward it to the cloud

- Cloud-based server: hosting an AI-based classification algorithm which outputs the probability of the user having or not $\mathrm{BC}$

As detailed in Fig. 9, the connectivity workflow of the aforementioned technology mentioned above works as follows: When a urine sample is introduced inside the device, the sensors perform a change in voltage depending on the nature of the chemical compounds present in the urine sample. Once the microprocessor has acquired the sensors' signal, the BLE module sends the sample's smellprint to the user's phone, where the software application is installed during the next 30 minutes. The app then sends this signal to the cloud via WiFi, where the AI-based classification AI algorithm is allocated. The algorithm classifies the sample with 95\% accuracy. The signal is now not processed on edge (as in the previous prototype) but on the cloud to allow better computational power and thus better model accuracy.

\section{Sample acquisition}

Urine samples were collected at the Southern Catalonia Institute of Oncology (Reus, Spain). Patient selection was carried out by a medical oncologist with experience in BC. Patients had locally advanced or metastatic BC (stages III and IV), thus 


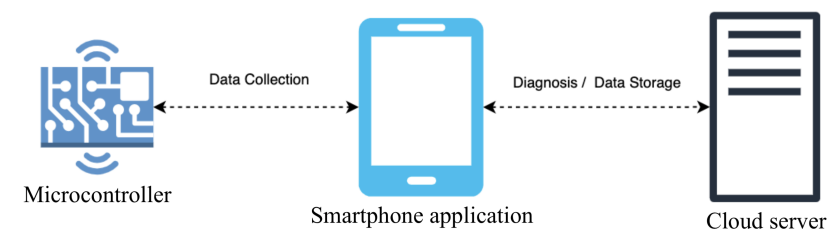

Figure 9. Connectivity workflow to gather data on edge and perform sample classification on the cloud

presenting a high tumor burden and therefore potentially an increased concentration of excreted metabolites in urine. Control subjects were invited to participate in the study as well. These samples were obtained from a random population segment. Urine samples were stored at $4^{\circ} \mathrm{C}\left(39.2^{\circ} \mathrm{F}\right)$ for no longer than 48 hours before analysis so that $80 \%$ of the intensity of their VOCs was preserved. ${ }^{57}$ However, according to ${ }^{58}$, there is no evidence of freezing the sample having a negative impact on its odor pattern.

Rovira i Virgili Institute of Medical Research's ethics committee approved the research. All research was performed following the provided good practice guidelines. Informed consent was obtained from all participants and/or their legal guardians. Only non-identifyable patient data was used.

\section{Experimental validation}

This model was trained with the following samples:

- 49 urine samples from control subjects

- 37 urine samples from BC patients

As shown in Fig. 10, urine samples have been experimentally tested using a gas chromatography-mass spectroscopy. This procedure aimed at determining whether the GC-MS was sensible enough to discriminate BC samples from control samples - thus obtaining evidence that there existed enough difference between the two classes of samples. Hence, the first step of sample classification was performed with GC-MS data. Once this hypothesis was proven, the same samples were analyzed with the aforementioned eNose and the resulting data were also classified using machine learning methods. The methodology implemented and the obtained results will be detailed as follows.

Please note that an eNose is based on a sensor array that responds differently according to specific VOCs. In fact, the sensor's response is not univocally correlated with the concentration of a single compound but rather a combination of the chemical information of a sample. However, because the sensitivity of each sensor for every VOC is different, it is possible to detect different overall compositions of samples and to classify them according to some macroscopic feature. ${ }^{41}$

\section{References}

1. US Preventive Services Task Force, 2009. Screening for breast cancer: US Preventive Services Task Force recommendation statement. Annals of internal medicine, 151(10), pp.716-236.

2. Nelson, H.D., Tyne, K., Naik, A., Bougatsos, C., Chan, B.K. and Humphrey, L., 2009. Screening for breast cancer: an update for the US Preventive Services Task Force. Annals of internal medicine, 151(10), pp.727-737.

3. Center for Disease Contorl (CDC), Health, United States, 2019, table 33

4. Feldstein, A.C., Perrin, N., Rosales, A.G., Schneider, J., Rix, M.M. and Glasgow, R.E., 2011. Patient barriers to mammography identified during a reminder program. Journal of Women's Health, 20(3), pp.421-428.

5. Jemal, Ahmedin, Elizabeth Ward, and Michael J. Thun. "Recent trends in breast cancer incidence rates by age and tumor characteristics among US women." Breast Cancer Research 9.3 (2007): 1-6.

6. Broeders, M., Moss, S., Nyström, L., Njor, S., Jonsson, H., Paap, E., Massat, N., Duffy, S., Lynge, E. and Paci, E., 2012. The impact of mammographic screening on breast cancer mortality in Europe: a review of observational studies. Journal of medical screening, 19(1 suppl), pp.14-25.

7. Blumen, H., Fitch, K. and Polkus, V., 2016. Comparison of treatment costs for breast cancer, by tumor stage and type of service. American health drug benefits, 9(1), p.23.

8. Desreux, J.A., 2018. Breast cancer screening in young women. European journal of obstetrics gynecology and reproductive biology, 230, pp.208-211.

9. World Health Organization, "WHO Position paper on mammography screening", 2014. 


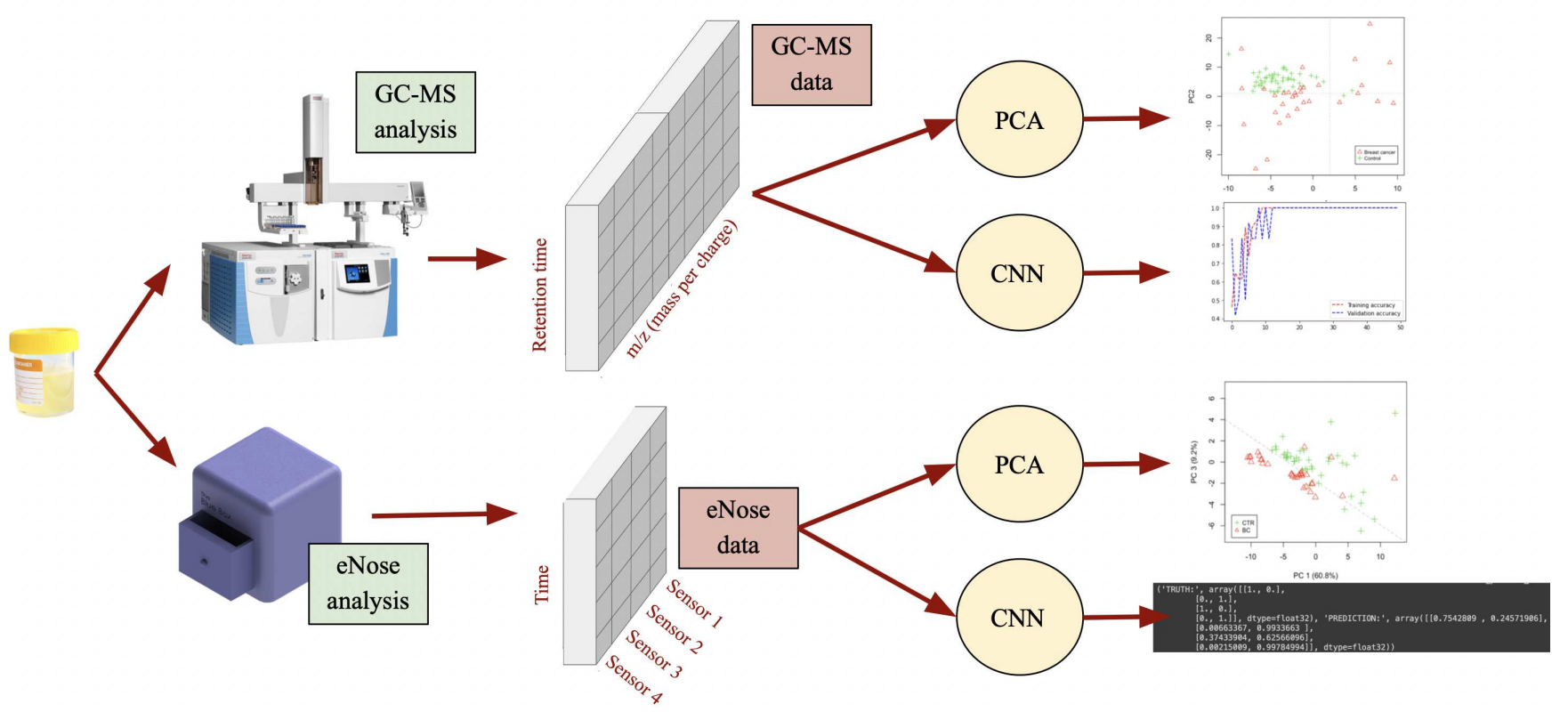

Figure 10. Design of the project. Upon collection, urine samples were analyzed using both an eNose and a GC-MS. Data resulting from the GC-MS analysis contain information relative to the $\mathrm{m} / \mathrm{z}$ of the VOCs found in the sample per unit of time (retention time). The output of the eNose analysis was a 2D array that was informative of the intensity of each sensors' reaction as a function of time. Finally, both datasets were input to a biostatistics-based classification (PCA) as well as an AI-based classification $(\mathrm{CNN})$.

10. "Disease burden and mortality estimates", World Health Organization, 2018.

11. Siu, A.L., 2016. Screening for breast cancer: US Preventive Services Task Force recommendation statement. Annals of internal medicine, 164(4), pp.279-296.

12. Sechopoulos, I., Suryanarayanan, S., Vedantham, S., D’Orsi, C.J. and Karellas, A., 2008. Radiation dose to organs and tissues from mammography: Monte Carlo and phantom study. Radiology, 246(2), pp.434-443.

13. Gøtzsche, P.C., 2004. The debate on breast cancer screening with mammography is important. Journal of the American College of Radiology, 1(1), pp.8-14.

14. Apantaku, L.M., 2000. Breast cancer diagnosis and screening. American Family Physician, 62(3), pp.596-602.

15. Podgornova, Y.A. and Sadykov, S.S., 2018, April. Detection of malignant breast tumors on the background of fibrocystic breast disease. In CEUR Workshop Proceedings (Vol. 2210, p. 177).

16. Malherbe, K. and Fatima, S., 2019. Fibrocystic breast disease.

17. Warner, E., Plewes, D.B., Hill, K.A., Causer, P.A., Zubovits, J.T., Jong, R.A., Cutrara, M.R., DeBoer, G., Yaffe, M.J., Messner, S.J. and Meschino, W.S., 2004. Surveillance of BRCA1 and BRCA2 mutation carriers with magnetic resonance imaging, ultrasound, mammography, and clinical breast examination. Jama, 292(11), pp.1317-1325.

18. Mango, V.L., Goel, A., Mema, E., Kwak, E. and Ha, R., 2019. Breast MRI screening for average-risk women: A monte carlo simulation cost-benefit analysis. Journal of Magnetic Resonance Imaging, 49(7), pp.e216-e221.

19. C. Guo, X. Li, M. Ye, F. Xu, J. Yu, C. Xie, X. Cao, M. Guo, Y. Yuan and S. Zheng, "Discriminating patients with early-stage breast cancer from benign lesions by detection of oxidative DNA damage biomarker in urine", Oncotarget, vol. 8, no. 32, 2017.

20. Y. Huang, Y. Li, Z. Luo and Y. Duan, "Investigation of biomarkers for discriminating breast cancer cell lines from normal mammary cell lines based on VOCs analysis and metabolomics", Royal Society of Chemistry Advances, vol. 6, no. 48, pp. 41816-41824, 2016. 
21. C. Wang, B. Sun, L. Guo, X. Wang, C. Ke, S. Liu, W. Zhao, S. Luo, Z. Guo, Y. Zhang, G. Xu and E. Li, "Volatile Organic Metabolites Identify Patients with Breast Cancer, Cyclomastopathy, and Mammary Gland Fibroma", Scientific Reports, vol. 4, no. 1, 2014.

22. C. Silva, R. Perestrelo, P. Silva, H. Tomás and J. Câmara, "Volatile metabolomic signature of human breast cancer cell lines", Scientific Reports, vol. 7, p. 43969, 2017.

23. Vignoli, A., Risi, E., McCartney, A., Migliaccio, I., Moretti, E., Malorni, L., Luchinat, C., Biganzoli, L. and Tenori, L., 2021. Precision Oncology via NMR-Based Metabolomics: A Review on Breast Cancer. International Journal of Molecular Sciences, 22(9), p.4687.

24. A. Dent, T. Sutedja and P. Zimmerman, "Exhaled breath analysis for lung cancer", Journal of Thoracic diseases, vol. 63, no. 2, pp. 164-168, 2013.

25. Persaud, Krishna, and George Dodd. "Analysis of discrimination mechanisms in the mammalian olfactory system using a model nose." Nature 299.5881 (1982): 352-355.

26. Asimakopoulos, A. D., et al. "Prostate cancer diagnosis through electronic nose in the urine headspace setting: a pilot study." Prostate cancer and prostatic diseases 17.2 (2014): 206-211.

27. H. Guerrero-Flores, T. Apresa-García, Ó. Garay-Villar, A. Sánchez-Pérez, D. Flores-Villegas, A. Bandera-Calderón, R. García-Palacios, T. Rojas-Sánchez, P. Romero-Morelos, V. Sánchez-Albor, O. Mata, V. Arana-Conejo, J. Badillo-Romero, K. Taniguchi, D. Marrero-Rodríguez, M. Mendoza-Rodríguez, M. Rodríguez-Esquivel, V. Huerta-Padilla, A. MartínezCastillo, I. Hernández-Gallardo, R. López-Romero, C. Bandala, J. Rosales-Guevara and M. Salcedo, "A non-invasive tool for detecting cervical cancer odor by trained scent dogs", BMC Cancer, vol. 17, no. 1, 2017.

28. B. Buszewski, T. Ligor, T. Jezierski, A. Wenda-Piesik, M. Walczak and J. Rudnicka, "Identification of volatile lung cancer markers by gas chromatography-mass spectrometry: comparison with discrimination by canines", Analytical and Bioanalytical Chemistry, vol. 404, no. 1, pp. 141-146, 2012.

29. R. Blatt, A. Bonarini and M. Matteuci, "Pattern Classification Techniques for Lung Cancer Diagnosis by an Electronic Nose", Springer-Verlag, vol. 4, no. 2, pp. 397-423, 2010.

30. M. Phillips, J. Beatty, R. Cataneo, J. Huston, P. Kaplan, R. Lalisang, P. Lambin, M. Lobbes, M. Mundada, N. Pappas and U. Patel, "Rapid Point-Of-Care Breath Test for Biomarkers of Breast Cancer and Abnormal Mammograms", PLoS ONE, vol. 9, no. 3, p. e90226, 2014.

31. J. Li, Y. Peng, Y. Liu, W. Li, Y. Jin, Z. Tang and Y. Duan, "Investigation of potential breath biomarkers for the early diagnosis of breast cancer using gas chromatography-mass spectrometry", Clinica Chimica Acta, vol. 436, pp. 59-67, 2014.

32. C. Burton and Y. Ma, "Current Trends in Cancer Biomarker Discovery Using Urinary Metabolomics: Achievements and New Challenges", Current Medicinal Chemistry, vol. 24, 2017.

33. C. Guo, X. Li, M. Ye, F. Xu, J. Yu, C. Xie, X. Cao, M. Guo, Y. Yuan and S. Zheng, "Discriminating patients with early-stage breast cancer from benign lesions by detection of oxidative DNA damage biomarker in urine", Oncotarget, vol. 8, no. 32, 2017.

34. G. Horvath, G. Järverud, S. Järverud and I. Horváth, "Human Ovarian Carcinomas Detected by Specific Odor", Integrative Cancer Therapies, vol. 7, no. 2, pp. 76-80, 2008.

35. L. Lavra, A. Catini, A. Ulivieri, R. Capuano, L. Baghernajad Salehi, S. Sciacchitano, A. Bartolazzi, S. Nardis, R. Paolesse, E. Martinelli and C. Di Natale, "Investigation of VOCs associated with different characteristics of breast cancer cells", Scientific Reports, vol. 5, no. 1, 2015.

36. van Keulen, K.E., Jansen, M.E., Schrauwen, R.W., Kolkman, J.J. and Siersema, P.D., 2020. Volatile organic compounds in breath can serve as a non-invasive diagnostic biomarker for the detection of advanced adenomas and colorectal cancer. Alimentary pharmacology therapeutics, 51(3), pp.334-346.

37. Antoce, A.O. and Namolosanu, I.O.A.N., 2011. Rapid and precise discrimination of wines by means of an electronic nose based on gas-chromatography. Rev. Chim, 62(6), pp.593-595.

38. Kishimoto, N. and Kashiwagi, A., 2019, May. Evaluation of Filtration on Volatile Compounds in Virgin Olive Oils using an Electronic Nose. In 2019 IEEE International Symposium on Olfaction and Electronic Nose (ISOEN) (pp. 1-3). IEEE.

39. G. Peng, M. Hakim, Y. Broza, S. Billan, R. Abdah-Bortnyak, A. Kuten, U. Tisch and H. Haick, "Detection of lung, breast, colorectal and prostate cancers from exhaled breath using a single array of nanosensors", British Journal of Cancer, vol. 103, no. 4, pp. 542-551, 2010. 
40. N. Peled, M. Hakim, P. Bunn, Y. Miller, T. Kennedy, J. Mattei, J. Mitchell, F. Hirsch and H. Haick, "Non-invasive Breath Analysis of Pulmonary Nodules", Journal of Thoracic Oncology, vol. 7, no. 10, pp. 1528-1533, 2012.

41. C. Di Natale, A. Macagnano, E. Martinelli, R. Paolesse, G. D’Arcangelo, C. Roscioni, A. Finazzi-Agrò and A. D’Amico, "Lung cancer identification by the analysis of breath by means of an array of non-selective gas sensors", Biosensors and Bioelectronics, vol. 18, no. 10, pp. 1209-1218, 2003.

42. A. D'Amico, G. Pennazza, M. Santonico, E. Martinelli, C. Roscioni, G. Galluccio, R. Paolesse and C. Di Natale, "An investigation on electronic nose diagnosis of lung cancer", Lung Cancer, vol. 68, no. 2, pp. 170-176, 2010.

43. Barlow, W.E., Chi, C., Carney, P.A., Taplin, S.H., D’Orsi, C., Cutter, G., Hendrick, R.E. and Elmore, J.G., 2004. Accuracy of screening mammography interpretation by characteristics of radiologists. Journal of the National Cancer Institute, 96(24), pp.1840-1850.

44. C. Weber, M. Cauchi, M. Patel, C. Bessant, C. Turner, L. Britton and C. Willis, "Evaluation of a gas sensor array and pattern recognition for the identification of bladder cancer from urine headspace", The Analyst, vol. 136, no. 2, pp. 359-364, 2011.

45. A. Roine, M. Tolvanen, M. Sipiläinen, P. Kumpulainen, M. Helenius, T. Lehtimäki, J. Vepsäläinen, T. Keinänen, M. Häkkinen, J. Koskimäki, E. Veskimäe, A. Tuokko, T. Visakorpi, T. Tammela, T. Sioris, T. Paavonen, J. Lekkala, H. Helle and N. Oksala, "Detection of smell print differences between nonmalignant and malignant prostate cells with an electronic nose", Future Oncology, vol. 8, no. 9, pp. 1157-1165, 2012.

46. J. Watson, "The tin oxide gas sensor and its applications", Sensors and Actuators, vol. 5, no. 1, pp. 29-42, 1984.

47. P. Mazzone, J. Hammel, R. Dweik, J. Na, C. Czich, D. Laskowski and T. Mekhail, "Diagnosis of lung cancer by the analysis of exhaled breath with a colorimetric sensor array", Thorax, vol. 62, no. 7, pp. 565-568, 2007.

48. R. Machado, D. Laskowski, O. Deffenderfer, T. Burch, S. Zheng, P. Mazzone, T. Mekhail, C. Jennings, J. Stoller, J. Pyle, J. Duncan, R. Dweik and S. Erzurum, "Detection of Lung Cancer by Sensor Array Analyses of Exhaled Breath", American Journal of Respiratory and Critical Care Medicine, vol. 171, no. 11, pp. 1286-1291, 2005.

49. S. Dragonieri, J. Annema, R. Schot, M. van der Schee, A. Spanevello, P. Carratú, O. Resta, K. Rabe and P. Sterk, "An electronic nose in the discrimination of patients with non-small cell lung cancer and COPD", Lung Cancer, vol. 64, no. 2, pp. 166-170, 2009.

50. O'Donovan P, Perrett CM, Zhang XH, Montaner B, Xu YZ, Harwood CA, McGregor JM, Walker SL, Hanaoka F, Karran P., "Azathioprine and UVA light generate mutagenic oxidative DNA damage". Science, vol. 309, pp. 1871-1874, 2005.

51. S. Brooks, D. Moore, E. Marzouk, F. Glenn and R. Hallock, "Canine Olfaction and Electronic Nose Detection of Volatile Organic Compounds in the Detection of Cancer: A Review", Cancer Investigation, vol. 33, no. 9, pp. 411-419, 2015.

52. List of MAK and BAT Values 2017: Permanent Senate Commission for the Investigation of Health Hazards of Chemical Compounds in the Work Area. Report 53, 1st ed. Berlin: WILEY-VCH Verlag GmbH and Co. KGaA, 2017, vol. 17, pp. 14-36.

53. "The PubChem Project", Pubchem.ncbi.nlm.nih.gov, 2018. [Online]. Available: https://pubchem.ncbi.nlm.nih.gov. [Accessed: 10-March- 2018].

54. "Human Metabolome Database", HMDB, 2018. [Online]. Available: http://www.hmdb.ca. [Accessed: 30- May- 2018].

55. P. Mochalski and K. Unterkofler, "Quantification of selected volatile organic compounds in human urine by gas chromatography selective reagent ionization time of flight mass spectrometry (GC-SRI-TOF-MS) coupled with head-space solid-phase microextraction (HS-SPME)", The Analyst, vol. 141, no. 15, pp. 4796-4803, 2016.

56. N. B rsan and U. Weimar, "Understanding the fundamental principles of metal oxide based gas sensors; the example of $\mathrm{CO}$ sensing with $\mathrm{SnO} 2$ sensors in the presence of humidity", Journal of Physics: Condensed Matter, vol. 15, no. 20, pp. 813-839, 2003.

57. T. Henriksen, P. Hillestrom, H. Poulsen and A. Weimann, "Automated method for the direct analysis of 8-oxo-guanosine and 8-oxo-2-deoxyguanosine in human urine using ultraperformance liquid chromatography and tandem mass spectrometry", Free Radical Biology and Medicine, vol. 47, no. 5, pp. 629-635, 2009.

58. S. Smith, H. Burden, R. Persad, K. Whittington, B. de Lacy Costello, N. Ratcliffe and C. Probert, "A comparative study of the analysis of human urine headspace using gas chromatography-mass spectrometry", Journal of Breath Research, vol. 2, no. 3, p. 037022, 2008. 


\section{Author contributions statement}

J.G.P. selected the patients and designed the sample collection protocol; J.G.B. and J.G.P. collected the 90 urine samples; J.G.B. and A.P.M. conceived the first experiment (biostatistics classification); J.G.B., M.S. and F.K. conceived the second experiment (ML classification); J.G.B. conducted the experiments; J.G.B., A.P.M. and J.G.P. analyzed the results of the first experiment; J.G.B., M.S., F.K. and M.K. analyzed the results of the second experiment. All authors reviewed the manuscript.

\section{Data availability statement}

The data that support the findings of this study are available from the corresponding author, J.G.B., upon reasonable request.

\section{Additional information}

J.G.B. owns stock in The Blue Box Biomedical Solutions, a company owning intellectual property related to this publication. All other authors declare no potential conflict of interest and no competing interests. 